\title{
GRAPH CLOSURES AND METRIC COMPACTIFICATIONS OF $N$
}

\author{
A. K. STEINER AND E. F. STEINER
}

Abstract. It is shown that all compactifications of the positive integers $N$ which have metrizable remainders are themselves metrizable. This is done by first proving that each Hausdorff compactification of a noncompact locally compact space is the graph closure in an appropriate space. It is then shown that any two compactifications of $N$ which have homeomorphic metrizable remainders are homeomorphic.

1. Introduction. In [6], we constructed compactifications with certain specified remainders as closures of graphs. We gave as a corollary to the main theorem a result of Aarts and van Emde Boas [1] which does not follow from it, but does from a generalized version which we will present. It will also follow that each Hausdorff compactification of a noncompact locally compact space is the graph closure in an appropriate space.

As an application, it will be shown that all compactifications of the positive integers $N$ which have metrizable remainders are themselves metrizable. An interesting result of von Neumann [5] which has recently been given short proofs in [4] and [7] will then be used to show that any two compactifications of $N$ which have homeomorphic metrizable remainders are homeomorphic.

2. Graph closures. All spaces considered are to be assumed Hausdorff. Often, when $(f, Y)$ is a compactification of $X, f[X]$ will be identified with $X$ and the embedding map will not be mentioned. If $Y$ is a compactification of $X, Y-X$ is the remainder of $X$ in $Y$. Whenever $f$ is a continuous mapping of $X$ into $Y, X$ is homeomorphic to the graph of $f$ and we will identify it with $X$. If $X$ is locally compact and noncompact, its one-point compactification is denoted by $X^{*}=X \cup\{\omega\}$ and $U(\omega)$ will be an open neighborhood of $\omega$ in $X^{*}$.

Theorem 1. Let $X$ be locally compact and noncompact and $K$ be contained in a compact space $T$. If there is a continuous mapping from $X$ into $T$ such that $K=\bigcap\left\{\operatorname{cl}_{T} f[U(\omega) \cap X]\right.$ : all $\left.U(\omega)\right\}$, then $X$ has a compactification $Y$ with $K$ as a remainder. The closure of the graph of $f$ in $X^{*} \times T$ is such a $Y$.

Received by the editors December 8, 1969.

AMS Subject Classifications. Primary 5453.

Key Words and Phrases. Compact spaces, compactifications, metric spaces, graph closures. 
Proof. The graph $G$ of $f$ in $X^{*} \times T$ is homeomorphic to $X$ and since $T$ is Hausdorff and $f$ continuous, no point $(x, t), t \neq f(x)$ is in $\bar{G}$. The condition on $K$ implies that $\{\omega\} \times K \subset \bar{G}$ and that $(\omega, t) \notin \bar{G}$ if $t \notin K$. Thus $\bar{G}$ is the desired compactification with remainder $\{\omega\} \times K$ homeomorphic to $K$.

Every compactification of a locally compact space can be obtained as a graph closure in the following way.

Corollary. If $(f, Y)$ is a compactification of a locally compact, noncompact space $X$, then $Y$ is equivalent to $(h, \bar{G})$ where $h$ is the mapping of $X$ onto $G$ which takes $x$ to $(x, f(x))$.

Proof. Let $k$ be the mapping of $Y$ onto $\bar{G}$ such that $k[f(x)]=h(x)$ for $x \in X$ and $k(y)=(\omega, y)$ for $y \in Y-f[X]$. Clearly $k$ is one-to-one and continuous on $f[X]$ and $Y-f[X]$. If a net $\left\{f\left(x_{\alpha}\right)\right\}$ in $f[X]$ converges to $y \in Y-f[X]$, then $\left\{x_{\alpha}\right\}$ must converge to $\omega$ in $X^{*}$. Thus $\left\{h\left(x_{\alpha}\right)\right\}$ converges to $(\omega, y)$ and hence $k$ is continuous on $Y$. Since $Y$ is compact and $\bar{G}$ is Hausdorff, $k$ is a homeomorphism such that $k \cdot f=h$. Therefore $(f, Y)$ and $(h, \bar{G})$ are equivalent. All the results in [6] now also follow.

3. Similar compactifications. Two compactifications of a space $X$ are equivalent if there is a homeomorphism between them which leaves $X$ pointwise fixed. We feel that for some purposes this notion distinguishes too many spaces; e.g. there are infinitely many nonequivalent two-point compactifications of $N$.

We will call two compactifications of $X$ similar if there is a homeomorphism between them which leaves $X$ fixed but not necessarily pointwise. We state the fairly obvious situation in $\beta X$ as

THEOREM 2. Two compactifications of $X$ are equivalent if and only if they give rise to identical decompositions of $\beta X$. They are similar if and only if there is an autohomeomorphism of $\beta X$ taking one decomposition onto the other.

It is easy to see that homeomorphic remainders need not imply similar compactifications; indeed, the compactifications may fail to be homeomorphic.

EXAMPLE 1. Let $X$ be an uncountable discrete space and consider it as the disjoint union of two uncountable sets and compactify $X$ by attaching one point to each of them. Now consider $X$ as the disjoint union of two sets, one of which is countable, and again attach one point to each. The two compactifications are not homeomorphic but the remainders are. 
To do this for $N$ is not quite so easy.

Example 2. Let $\varepsilon$ be an infinite and maximal family of infinite subsets of $N$ such that the intersection of any two is finite. As in 5I of [3], let $D=\left\{P_{E}: E \in \mathcal{E}\right\}$ be a new set of distinct points and define $Y^{\prime}=N \cup D$ with the following topology: the points of $N$ are isolated, while a neighborhood of $P_{E}$ is any set containing $P_{E}$ and all but a finite number of points of $E$. Let $F \in \mathcal{E}$ and define $Z^{\prime}=Y^{\prime}$ $-\left\{P_{F}\right\}$. Both $Y^{\prime}$ and $Z^{\prime}$ are locally compact, noncompact Tychonoff spaces and thus have one-point compactifications $Y$ and $Z$, respectively. Both $Y-N$ and $Z-N$ are homeomorphic to the one-point compactification of the discrete space of cardinality $c$. Since $Y$ and $Z$ each have exactly one point with an uncountable base, any homeomorphism of $Z$ onto $Y$ would take $Z^{\prime}$ onto $Y^{\prime}$. But $F$ is an infinite discrete open and closed subset of $Z^{\prime}$ and no infinite discrete subset of $Y^{\prime}$ is both open and closed. Thus $Y$ and $Z$ are nonhomeomorphic compactifications of $N$ with homeomorphic remainders.

Since the elements of $N$ are isolated in any compactification, it follows that any two of its compactifications which are homeomorphic are similar.

In general this is not so, even given that the remainders are homeomorphic.

EXAmple 3. Consider the locally compact subspace of the reals given by $X=(0,1) \cup[(1,2)-\{1+1 / n: n=1,2,3, \cdots\}] \cup(2,3)$. Let $f$ and $g$ be continuous mappings of $X$ onto the closed interval $[-1,1]$ defined by

$$
\begin{array}{rlrl}
f(x) & =\sin \frac{\pi}{x}, \quad 0<x<1, \quad g(x) & =0,0<x<2, \\
& =0, \quad 1<x<3, & & =\sin \frac{\pi}{3-x}, \quad 2<x<3 .
\end{array}
$$

If $F$ and $G$ denote the graphs of $f$ and $g$ in $[0,3] \times[-1,1]$, then $\bar{F}$ and $\bar{G}$ are homeomorphic compactifications of $X$. Also, $\bar{F}-F$ is homeomorphic with $\bar{G}-G$. However, there is no homeomorphism of $\bar{F}$ onto $\bar{G}$ which takes $F$ onto $G$.

4. Metric compactifications of $N$. We saw in Example 2 that a homeomorphism between remainders is not sufficient to show that compactifications of $N$ are similar. If the remainders are metrizable, it is sufficient. We will first prove

THEOREM 3. If $(f, Y)$ is a compactification of $N$ with a metrizable remainder, then $Y$ is metrizable. 
Proof. Let $F$ be the extension of $f$ to $\beta N$ and $f^{*}$ its restriction to $\beta N-N$. Since $X=Y-f[N]$ is a compact metric space, it may be embedded in $P=\Pi\left\{I_{i}: i=1,2, \cdots\right\}$ where $I_{i}=[0,1]$. If $\pi_{i}$ denotes the projection of $P$ onto $I_{i}$, then $\pi_{i} \circ f^{*}$ is a continuous mapping of $\beta N-N$ into $[0,1]$ and can be continuously extended to a mapping $g_{i}$ of $\beta N$ into $[0,1]$. Moreover, since $N$ is discrete and countable, it may be assumed that $g_{i}(i) \neq g_{i}(j)$ for all $j<i$. Let $g$ be the mapping of $\beta N$ into $P$ defined as $g(x)_{i}=g_{i}(x)$. Then $g$ is continuous, one-to-one on $N$ and $g|\beta N-N=F| \beta N-N$. If $U$ is a cofinite subset of $N$, then $X=g[\beta N-N] \subset g\left[\operatorname{cl}_{\beta N} U\right] \subset \operatorname{cl}_{P} g[U]$. Thus $X \subset \cap\left\{\operatorname{cl}_{P} g[U]: U\right.$ cofinite subset of $N\}$. Since $\beta N$ is compact, $\operatorname{cl}_{P} g[U] \subset g[\beta N]$ for each cofinite $U \subset N$. If $y \in g[\beta N]-X$, then $y=g(n)$ for some $n \in N$ and $y \notin g[\beta N-\{n\}]$ since $g$ is one-to-one on $N$. Thus $y \notin \operatorname{cl}_{P} g[N-\{n\}]$ and $X=\cap\left\{\operatorname{cl}_{P} g[U]: U\right.$ cofinite $\left.\subset N\right\}$.

The graph $G$ of $g$ lies in $N^{*} \times P$, and by Theorem $1, \bar{G}$ is a compactification of $N$ with remainder $X$. Since $N^{*}$ and $P$ are metrizable so is $\bar{G}$. Define $e(n)=(n, g(n))$ and let $E$ be the extension of $e$ from $\beta N$ onto $\bar{G}$. We will show that $(e, \bar{G})$ and $(f, Y)$ give the same decomposition of $\beta N$. Suppose $q \in \beta N-N, E(q)=(\omega, x) \in \bar{G}-G$ and $F(q)=y \in X$. If $x \neq y$, then there are disjoint open sets $V, W \subset P$ such that $x \in V$ and $y \in W$. Since $F(q)=f^{*}(q)=g(q)$ and $g$ is continuous, $g^{-1}[W]$ is an open neighborhood of $q$ in $\beta N$. Also $E^{-1}\left[N^{*} \times V\right]$ is an open neighborhood of $q$ in $\beta N$. Let $n \in g^{-1}[W] \cap E^{-1}\left[N^{*} \times V\right] \cap N$. Then $E(n)$ $=(n, g(n))$ implies that $g(n) \in V$. Since $g(n) \in W$ we get the contradiction that $V \cap W \neq \varnothing$. Thus $x=y$. Therefore, $(e, \bar{G})$ and $(f, Y)$ are equivalent compactifications and since $\bar{G}$ is metrizable, so is $Y$.

TheOREM 4. Compactifications of $N$ with homeomorphic metrizable remainders are similar.

Proof. Let $\left(f_{1}, Y_{1}\right)$ and $\left(f_{2}, Y_{2}\right)$ be compactifications of $N$ with remainders homeomorphic to a compact metric space $X$. Following the proof of Theorem $3,\left(f_{1}, Y_{1}\right)$ and $\left(f_{2}, Y_{2}\right)$ are equivalent to $\left(e_{1}, \bar{G}_{1}\right)$ and $\left(e_{2}, \bar{G}_{2}\right)$, respectively, where $\bar{G}_{1}$ and $\bar{G}_{2}$ are subsets of $N^{*} \times P$ and have remainders $\{\omega\} \times X$ as a common set of cluster points. By a result of von Neumann [5] (cf. [4] or [7]), there is a permutation $\pi$ on $N$ such that $d\left(e_{1}(n), e_{2}(\pi(n))\right) \rightarrow 0$. If $h$ is the mapping on $\bar{G}_{1}$ defined as $h\left(e_{1}(n)\right)=e_{2}(\pi(n)), n \in N$, and $h(\omega, x)=(\omega, x), x \in X$, then $h$ is a homeomorphism onto $\bar{G}_{2}$ which takes $e_{1}[N]$ onto $e_{2}[N]$. Thus $\left(e_{1}, \bar{G}_{1}\right)$ and $\left(e_{2}, \bar{G}_{2}\right)$ are similar. It follows that $\left(f_{1}, Y_{1}\right)$ and $\left(f_{2}, Y_{2}\right)$ are also similar.

It is known [2] that any zero-set in $\beta N-N$ is homeomorphic to $\beta N-N$. If two zero-sets in $\beta N-N$ are open, there is a permutation 
of $N$ whose extension to $\beta N$ will take one onto the other $[3,6 \mathrm{~S}]$. We show this for nonopen zero-sets in the following

COROLlaRY. For any two nonopen zero-sets in $\beta N-N$, there is an autohomeomorphism of $\beta N$ which takes one onto the other.

Proof. If $Z$ and $Z^{\prime}$ are any two nonopen zero-sets in $\beta N-N$, then there are continuous mappings $f$ and $g$ of $\beta N$ onto $[0,1]$ such that $Z=g^{-1}[0]$, and $Z^{\prime}=f^{-1}[0]$.

The restriction of $g$ to $\beta N-N$ gives a decomposition of $\beta N-N$ which together with singletons from $N$ is an upper semicontinuous decomposition of $\beta N$. The quotient of this decomposition is a compactification $Y$ of $N$ with remainder $[0,1]$. Similarly, $f$ produces a compactification $Y^{\prime}$ of $N$ with remainder $[0,1]$. By Theorem $4, Y$ is similar to $Y^{\prime}$ and so by Theorem 2, there is an autohomeomorphism of $\beta N$ which takes $Z$ onto $Z^{\prime}$.

\section{REFERENCES}

1. J. M. Aarts and P. van Emde Boas, Continua as remainders in compact extensions, Nieuw Arch. Wisk. (3) 15 (1967), 34-37. MR 35 \#4885.

2. W. W. Comfort and S. Negrepontis, Homeomorphs of three subspaces of $\beta N \backslash N$, Math. Z. 107 (1968), 53-58. MR 38 \#2739.

3. L. Gillman and M. Jerison, Rings of continuous functions, The University Series in Higher Math., Van Nostrand, Princeton, N. J., 1960. MR 22 \#6994.

4. P. R. Halmos, Permutations of sequences and the Schröder-Bernstein theorem, Proc. Amer. Math. Soc. 19 (1968), 509-510. MR 37 \#2179.

5. J. von Neumann, Charakterisierung des Spektrums eines Integraloperators, Hermann, Paris, 1935.

6. A. K. Steiner and E. F. Steiner, Compactifications as closures of graphs, Fund. Math. 63 (1968), 221-223. MR 38 \#6546.

7. J. A. Yorke, Permutations and two sequences with the same cluster set, Proc. Amer. Math. Soc. 20 (1969), 606.

Iowa State University, Ames, Iowa 50010 